\title{
Beyond accessibility? Toward an on-line and memory-based model of framing effects
}

\author{
Matthes, Jörg
}

\begin{abstract}
This theoretical article investigates the effects of media frames on individuals' judgments. In contrast to previous theorizing, we suggest that framing scholars should embrace both, on-line and memory-based judgment formation processes. Based on that premise, we propose a model that distinguishes between two phases of framing effects. Along the first phase, the media's framing contributes to the formation of an on-line or a memory-based judgment. The second phase describes six hypothetical routes for the stability or the change of these judgments: maintenance, readjustment, crystallization, inoculation, persuasion, and attenuation. At the heart of our model, we try to extract predictors for each of those routes. Finally, the implications of the proposed model for future framing research are discussed.
\end{abstract}

DOI: https://doi.org/10.1515/COMMUN.2007.003

Posted at the Zurich Open Repository and Archive, University of Zurich ZORA URL: https://doi.org/10.5167/uzh-94829

Journal Article

Published Version

Originally published at:

Matthes, Jörg (2007). Beyond accessibility? Toward an on-line and memory-based model of framing effects. European Journal of Communication Research, 32(1):51-78.

DOI: https://doi.org/10.1515/COMMUN.2007.003 


\title{
Beyond accessibility? Toward an on-line and memory-based model of framing effects
}

JÖRG MATTHES

\begin{abstract}
This theoretical article investigates the effects of media frames on individuals' judgments. In contrast to previous theorizing, we suggest that framing scholars should embrace both, on-line and memory-based judgment formation processes. Based on that premise, we propose a model that distinguishes between two phases of framing effects. Along the first phase, the media's framing contributes to the formation of an on-line or a memory-based judgment. The second phase describes six hypothetical routes for the stability or the change of these judgments: maintenance, readjustment, crystallization, inoculation, persuasion, and attenuation. At the heart of our model, we try to extract predictors for each of those routes. Finally, the implications of the proposed model for future framing research are discussed.
\end{abstract}

Keywords: framing, on-line judgments, memory-based judgments, accessibility, persuasion, media effects

\section{Introduction}

How do people make sense of politics and what role does mass communication play in that process? This fundamental question of media effects research has fascinated scholars since Walter Lippmann's (1922) seminal book Public opinion. In recent years, communication researchers have become increasingly interested in the concept of framing as a means to describe the influence of the mass media on people's thoughts and attitudes (Entman, 1993; Iyengar, 1991; McCombs and Ghanem, 2001; B. Scheufele, 2004). Although a lion's share of framing research is devoted to the description of media frames (e.g., Igartua, Cheng, and Muñiz, 2005) or journalistic frames (e.g., B. Scheufele, 2006), there is also an increasing number of studies examining the 
effects of media frames on the public's attitudes (e.g., de Vreese, 2004; de Vreese and Boomgaarden, 2003; Cappella and Jamieson, 1997; Nabi, 2003; Price, Tewksbury, and Powers, 1997; Shen, 2004). The basic idea of the framing effects perspective is that by selecting some information and highlighting it to the exclusion of other information, framing can shape the audience's interpretations of issues, candidates, and events. As recent studies have convincingly demonstrated, framing can have a profound impact on the way individuals understand issues (e. g., Nabi, 2003; Shen, 2004). In fact, most research asserts that news framing makes some considerations more salient to audiences, and, therefore, these considerations are more likely to be used in subsequent evaluations (Iyengar, 1991; Nabi, 2003; Price et al., 1997; B. Scheufele, 2004; Shen, 2004).

In this article, we focus on attitudinal effects of media frames, i. e., mere knowledge effects will not be discussed here (for a comprehensive review about framing effects on individuals' schemata, see B. Scheufele, 2004). Taken together, framing effects research encompasses a rich empirical literature, and it offers a theoretical model for our understanding of how the news influences individuals' attitudes. However, what all these lines of research share, we argue, is the general proposition of a memory-based judgment formation process (Cappella and Jamieson, 1997; Druckman and Nelson, 2003; Nabi, 2003). More specifically, the idea of accessibility can be considered as the foundation of a memory-based model of information processing (D. Scheufele, 2000). That means, in expressing their opinions, individuals draw on the information that comes to their minds at the time a judgment is called for (Hastie and Park, 1986). Although these accounts of framing effects contribute to the accumulation of highly valuable knowledge in the field, we lack empirical studies and theoretical models that go beyond the idea of accessibility-based judgment formation. Conceptually, the memory-based model contrasts with an on-line model which holds that people do not search their memory for relevant information in order to construct a judgment (Hastie and Park, 1986; Lavine, 2002; Shrum, 2004). According to the on-line model, judgments are formed right away when the information is encountered. In other words, people form their attitudes at the time they initially process the information. Because an on-line judgment has been made during message presentation, people can rapidly retrieve this previously formed judgment at a later point in time. In contrast, memory-based judgments are computed after message presentation. Thus, they depend on the information that can be recalled at the time a judgment is called for (Hastie and Park, 1986; Mackie and Asuncion, 1990). 
Conceptually, we believe that "both processes - memory-based and on-line - are needed to explain framing effects" (Cappella and Jamieson, 1997: 71). Therefore, we suggest a process model of framing effects in this contribution that heavily draws on the idea of both, on-line and memory-based judgments.

\section{Framing effects research}

There are essentially two pathways to the study of framing effects that have received scholarly attention (Druckman, 2002). The first type, the so-called equivalency framing, examines how differently phrased but logically equivalent scenarios impact the decision making of individuals (Kahneman and Tversky, 1984). Although Kahneman and Tversky (1984) are often cited as pioneers in the field of framing (e. g., D. Scheufele, 2000), scholars in mass communication have preferred the second type of framing effects: the emphasis framing approach (Druckman, 2002). Emphasis framing does not refer to logically equivalent versions of a message but to real news stories that differ in their salience of several considerations (Entman, 1993). Because of its inherent relevance for communication research, we will focus on emphasis framing effects in this paper. In doing so, we are especially interested in the effects of emphasis frames on attitudes.

Without a doubt, it has been the original work by Iyengar (1991) that has fueled research on emphasis framing like never before. Iyengar (1991) examined the impact of news framing on the way individuals ascribe responsibility for several issues. At the heart of his research, he distinguishes between the episodic and thematic framing of issues. When news is framed episodically, social issues are constructed around specific instances and individuals. In contrast, thematic framing emphasizes broader trends or backgrounds about issues. In a series of experimental studies, Iyengar (1991) found some evidence that subjects shown episodic reports were less likely to consider society responsible, and subjects shown thematic reports were less likely to consider individuals responsible. These effects are explained by the accessibility of considerations. Iyengar (1991) states that viewers' explanations of issues critically depend on the particular reference points furnished in media presentations. In other words, framing effects are resulting from an accessibility bias (Iyengar, 1991). When forming a judgment, individuals do not draw on all possible information they ever encountered, in contrast, they rely on information made accessible by media coverage: "[T]he theory is that information that can be more easily retrieved from memory tends to dominate judgments, opinions, and decisions" (Iyengar, 1991: 130-131). 
This line of reasoning is supported by a plethora of studies that have examined the impact of news framing on individuals' thoughts and cognitive responses (Nabi, 2003; Price et al., 1997; Shen, 2004; Valkenburg, Semetko, and de Vreese, 1999). These studies implicitly or explicitly assume that media frames exert their influence on attitudes by shaping the accessibility of cognitions in the first place (B. Scheufele, 2004). The most elaborate model of accessibility-based framing effects stems from Price and Tewksbury (1997). Price and Tewksbury posit that the news media determine what bits of information are activated when people are called to make a judgment about an issue. This idea is based on an associative network model of human memory which assumes that knowledge is organized as an associated web of cognitive units or nodes. Within this network, the activation of one unit can spread through the network to interconnected units leading to the activation of related concepts. At any single point in time, only a small part of the knowledge store is subject to active thought. First, there are nodes that always ready to be activated because they have a high baseline excitation level (chronically accessible concepts). Moreover, there are salient attributes of the current situation which render other concepts that are applicable to that situation (temporarily accessible concepts). As known from psychological priming research, both recency and frequency of activation contribute to a construct's temporary accessibility (Higgins, 1996). When individuals read or watch news, concepts are activated which have the highest excitation level. When these concepts are judged as relevant for the situation at hand, they are used in evaluations. More specifically, Price and Tewksbury (1997) distinguish between applicability and accessibility effects: During message processing, salient stimuli of media coverage activate certain concepts. This is what they call an applicability effect. Once activated, these concepts retain some residual activation potential. When an evaluation is called for, once activated concepts compete with other chronically or temporarily activated concepts. Among those competing concepts, the one with the highest excitation potential is more likely to be used in subsequent evaluations (accessibility effect). A prerequisite for any accessibility effect is that the concepts are deemed as relevant to the situation at hand.

In line with Price and Tewksbury's model, the results of a vast number of other studies give convincing support for these kinds of effects (to name only a few de Vreese, 2004; de Vreese and Boomgaarden, 2003; Shen, 2004). However, Nelson, Clawson, and Oxley (1997) provide evidence that accessibility-based framing effects may include more elaborate processes than previously assumed. The authors state that framing effects work through a psychological process in which 
individuals think about the importance of relevant considerations. Although Nelson and colleagues stress that their model goes beyond accessibility effects, it is, as Druckman and Nelson (2003: 732) state, "based largely on the memory-based model". Finally, we can find an accessibility-based reasoning in the field of second-level agenda setting (McCombs and Ghanem, 2001).

As should be apparent from the previous section, most theorizing about framing effects draws on a memory-based model (for the same conclusion see Cappella and Jamieson, 1997; Druckman and Nelson, 2003; Nabi, 2003) - "with accessibility as the main theoretical explanation" (Gross and D'Ambrosio, 2004: 3). Although sometimes implicitly, framing scholars seem to agree that individuals - more or less deliberately - draw on the information that comes to their minds at the time of judgment. Of course, accessibility is a vital term for media effects in general, and for framing effects in particular (Iyengar, 1991). Without doubt, accessibility-based models of judgment formation have made highly significant contributions to various fields of scholarship. In the field of framing research, Price and Tewksbury's model represents a significant step in the direction of specifying the exact nature of framing effects, and the model is supported by a good deal of empirical evidence. However, social psychological research informs us that some judgments are formed on-line without a memory search for judgment-relevant information. Later on, these on-line judgments are merely retrieved, and no memory-based processes are involved in media effects. Although some authors have pointed to the necessity of considering both judgment types for framing effects research (Cappella and Jamieson, 1997; Druckman and Nelson, 2003), the predominance of memory-based reasoning is striking. Put more bluntly, "the memory-based model reduces media effects to accessibility effects" (Cappella and Jamieson, 1997: 72).

Moreover, previous research has mainly applied a tabula rasa model of framing effects without the consideration of already existing online or memory-based judgments. Nearly all studies have worked with a post-test experimental design where individuals are exposed to a news frame, and then they immediately report their opinions. In most instances, the information is only presented once, and a time component is rarely taken into account. That means framing scholars often have been inattentive to on-line or memory-based judgments that exist prior to the presentation of a news frame (D. Scheufele, 2000). In other words, although such experiments offer highly valuable and rich insights for framing theory, they are unable to model the whole process of framing effects, from the forming of an initial on-line or memory-based judgment to the change of this judgment in the course 
of subsequent media coverage. Clearly, a theoretical model is needed that reconciles the differences among both judgment types, specifies the circumstances when one or the other mechanism is more likely to operate, and describes how both judgment types can be changed.

Based on these insights, we posit four preliminary premises for a model of attitudinal framing effects. Our first premise is that both, on-line and memory-based judgment formation processes, should be part of such a model. This is not to say that previous memory-based models and studies are not useful, in contrast, the available findings are fully compatible with the account we propose in this paper. Second, an integrative model of framing effects has to include a timecomponent, that is, it has to predict which effects can occur in a long-term perspective (de Vreese, 2004). Third, on-line and memorybased judgments can be predicted for individuals with different predispositions (Lavine, 2002; McGraw, Hasecke, and Conger, 2003). Fourth and more importantly, we can expect different patterns of change and different predictors of change for on-line compared to memory-based judgments. In order to develop a more comprehensive understanding of these two processes, we will shortly discuss on-line and memorybased judgments in the following section. On these grounds, we will then turn to a model of framing effects.

\section{On-line and memory-based judgment formation}

The distinction between on-line and memory-based judgment formation is based on differences in the sources of information that are entered as input into the judgment operator (Hastie and Park, 1986). According to the on-line model, judgments are formed when the information is encountered. In other words, people form opinions at the time they initially process the information. If a judgment is called for at a later time, people just retrieve a previously formed judgment without a thorough memory search for judgment-relevant information. Thus, memory and judgment will not necessarily be correlated for on-line judgments. In contrast, the memory-based model holds that judgments are formed based on the information that people can recall from memory at the time a judgment is needed. This means, people do not attempt to form a judgment during initial information processing. Thus, as the name implies, memory-based judgments are based on whatever information is available at the time of judgment. Unlike on-line judgments, judgment and memory for judgment-relevant information should be correlated. Moreover, memory-based judgments should produce recency effects for recall. A recency effect for recall means that not the initial information is remembered but the informa- 
tion that has been recently encountered. The reason is that recently provided information has a higher accessibility, and can thus be better remembered (Higgins, 1996).

In a seminal study, Hastie and Park (1986) aimed at exploring the conditions for judgment tasks that would induce on-line and memorybased inferences. In their experimental setting, subjects in the on-line condition were told they would be playing the role of a personnel officer and making judgments about job-candidates. More precisely, subjects had to rate candidates according to central attributes required for the job. With this judgment task in mind, they listened to a taperecorded conversation between two job candidates. After listening to the tape, subjects rated the person's suitability for the job (judgment) and recalled everything they could remember (memory). In the memory-based condition, in contrast, subjects were told nothing about the job suitability task before they listened to the tape, i. e. they didn't know what kind of information they would need at a later point in time. As predicted, surprising the subjects with an unexpected judgment task resulted in a high memory-judgment correlation and, in contrast, preparing subjects for the task led to a non-significant correlation. This effect has been replicated in numerous studies in social psychology (to name a few, Mackie and Asuncion, 1990; Hamilton, Sherman, and Maddox, 1999; Tormala and Petty, 2001).

Both judgment types have also been used in communication research (e.g., Huang, 2000; Shrum, 2004) and political psychology (e. g., Lavine, 2002; McGraw et al., 2003). According to McGraw (2000: 813), "the identification and empirical evaluation of these two models of opinion formation are among the most impressive contributions of the cognitive approach". A very prominent example is the memory-based model of public opinion formation proposed by Zaller (1992). Zaller argues that people possess multiple, conflicting considerations relevant to a given issue. More precisely, they form their attitudes by averaging all considerations that are accessible at the time of judgment. An important implication of this model is that people are unlikely to have stable attitudes; i. e., the attitude depends on which considerations happen to come to the top of the head (Wilson and Hodges, 1992). In contrast to the memory-based model of opinion formation, the on-line model holds that people form and maintain a running evaluation counter of political objects (e.g., issues, candidates). When the individual encounters new information this evaluation counter is brought into working memory, and the stored judgment will be retrieved. The consequence is, as Lodge et al. (1989: 401) put it, that "people can often tell you how much they like or dislike a book, movie, candidate, or policy but not be able to recount 
the specific whys and wherefores for their overall evaluation". From the perspective of framing effects research, the on-line model has an important implication: People need not to maintain large memory stores of knowledge when making judgments (Druckman and Lupia, 2000). Moreover, the notion of on-line political judgments is well supported by a series of experiments (e.g., Lodge et al., 1989; McGraw Hasecke, and Conger, 2003). Taken together, on-line and memory-based processes have proven to be indispensable ingredients for a coherent theory of judgment formation (Lavine, 2002; McGraw et al., 2003). The theoretical account we suggest in this paper attempts to build upon this theorizing.

\section{A process model of framing effects}

Rather than taking a general perspective on attitude construction and change, we aim at providing a framing effects account. We believe that individuals either form a judgment based on accessible considerations (after message reception) or on a spontaneous evaluation of the information (during message reception). In both cases, however, we refer to media frames as the crucial independent variable. We do not focus on other possible forms of influence such as emotional media content, interpersonal communication or group membership. From a theoretical perspective, of course, any model of framing effects that goes beyond knowledge effects must be a model of attitude construction and change. However, our model shall be general enough to incorporate all kinds of issue-specific frames. Therefore, the focus of our model is on these processes and not on specific content characteristics or specific media frames. In order to generate the model in a clear manner, we derive a set of theoretical key-propositions in a first step. This is followed by an in-depth description of our model in a second step.

\section{Proposition 1}

First of all, we do not differentiate between judgments and attitudes. Traditionally, attitudes have been conceptualized as stable, evaluative constructions that are hard to change. Judgments, in contrast, are usually understood as short-term evaluations. Over the past decades of social psychological research, however, this distinction has become increasingly blurred (Schwarz, 2000). We adopt an understanding of attitudes as evaluative judgments (Schwarz, in press) that need not to be enduring personal dispositions. Put more bluntly, "the psychology of attitudes is simply the psychology of evaluative judgment" (Schwarz, 
2000: 168). In the social psychological literature, the so-called file-drawermodel (i. e., attitudes are stable and can be retrieved) is often contrasted with the so-called attitude-as-construction-model (i. e., attitudes are constructed based on accessible information). In our understanding, both models hold true and are adequate for different situations. The extent to which an attitude is retrieved from memory (as it stands) or to which it is built upon accessible considerations is an empirical issue. Thus, when we acknowledge that attitudes can either be stored and retrieved, or created on the spot, we need not to differentiate between judgments and attitudes. This reasoning also corresponds to the on-line/memory-based literature (Mackie and Asuncion, 1990): An on-line judgment can be defined as an attitude that is initially formed and can be easily retrieved later on (see also Fazio, 2000). A memory-based judgment, in contrast, can be understood as an attitude that is formed depending on the considerations accessible at the time of judgment reporting. Based on the understanding of both terms by Schwarz (2000), attitude and judgment will be used interchangeably in the following sections.

\section{Proposition 2}

Taking a process-perspective, it is important to distinguish between several time phases. First, the most important differentiation is between the time of frame reception and the time of attitude reporting. Only when these two time points are taken into account, can the type of judgment be explained to full extent. Second, it is important to distinguish between judgment formation about a new issue (no prior judgment) and about an established issue where judgments have been formed before. Later on, we will refer to this latter distinction as the two phases of framing effects. In theoretical terms, this distinction helps us to understand how the media impacts judgments and attitudes for novel and for established issues.

\section{Proposition 3}

We assume that, for every new issue (i. e., no retrievable prior judgment), individuals either use the on-line or the memory-based judgment formation approach (for this argument, see Hastie and Park, 1986). Applying an on-line approach, individuals will immediately form a judgment during media reception. This judgment can be easily retrieved at a later point in time. When applying a memory-based strategy, in contrast, people will not form a judgment during media reception; however, when they are asked later what they think about an issue or candidate, they will construct a judgment based on the recall of information received 
from media coverage. To reiterate, it is assumed that it is indeed possible not to form a judgment during media reception (see also proposition four). Without forming a judgment, however, it is still possible that individuals casually receive and learn information that can be accessed later on. To give an example, it is plausible that individuals do not always form a judgment about any new issue in the news. This would be too time-consuming and demanding. Especially for low-involvement issues, people rather passively monitor the news without coming up immediately with a judgment about what has happened. In more general terms, Hastie and Park (1986) themselves have discussed this important question in their seminal studies, and empirical research has also shown that individuals do not always form judgments when they encounter news messages (Zaller, 1992). Also, a large part of attitude theory (Schwarz, in press) builds on this premise.

As we have learned from previous studies, whether individuals form an on-line or a memory-based judgment depends on the processing goal. When there is the processing goal to form a judgment, an on-line judgment will result. When there is no such goal, no judgment will be formed at the time of message reception. As will be outlined later on, depending on motivational variables processing goals can change.

\section{Proposition 4}

In line with the literature on on-line and memory-based judgments, we treat both judgment types as two distinct forms. As stated in proposition three, people either have an on-line or a memory-based judgment. Nevertheless, we assume that we do not always have pure judgments. Online and memory-based judgments can also be in a kind of synergistic relationship. However, we propose that there should still be a dominating judgment type. A synergistic view would mean that the more online processing occurs during message reception, the less retrospective memory-based processing is needed at a later point in time (Hamilton et al., 1999). This implies that, for instance, individuals with an on-line judgment might also retrieve additional information stored in memory (i. e., a memory-based process), and combine this additional information with the existing judgment. However, this is not plausible for memorybased judgments. In principle, individuals will always try to access an on-line judgment when they have one. So only when there is no on-line judgment available, individuals must form a memory-based one. Therefore, it is not plausible that individuals will additionally retrieve an online judgment in the memory-based mode. Put differently, why should an individual create a time-consuming memory-based judgment when an 
on-line judgment is available? Therefore, a synergistic relationship is only possible for on-line judgments.

From a theoretical point of view the crucial point is, as stated above, that there always should be a dominating judgment type. Clearly, there is a threshold for when a memory-based judgment turns into an on-line judgment, but this is an empirical question. Furthermore, as we can learn from other dual-process theories (see Chaiken and Trope, 1999), clear distinctions enable clear predictions. Without a dominating judgment type it would be extremely difficult to empirically distinguish between an on-line and a memory-based judgment. In fact, any interpretation of a judgment type would be circular, as we have no means to derive clear hypotheses for a 'mixture-judgment'. However, assuming dominant judgment types, we have means to derive straight hypotheses in order to empirically differentiate them, as our next proposition explains.

\section{Proposition 5}

(Dominant) on-line and memory-based judgments differ in their characteristics, and these characteristics can be measured empirically. First, there is an array of empirical evidence that on-line judgments are held with greater attitude strength (Matthes, Wirth, and Schemer, 2007, Hamilton, Sherman, \& Maddox, 1999; Lavine, 2002). Second, individuals with on-line judgments should be faster to report their attitudes compared to individuals who hold memory-based judgments (Matthes et al., 2007; Mackie and Asuncion, 1990). Third, and more importantly, we can expect that memory and judgment will not necessarily be correlated for on-line judgments (Hastie and Park, 1986). However, unlike on-line judgments, judgment and memory should be correlated for memorybased judgments. Beside these classic variables, a recently developed scale for the measurement of on-line and memory-based judgment in surveys (Matthes et al., 2007) offers new possibilities for non-experimental research.

\section{Proposition 6}

Once established on-line or memory-based judgments can change in the course of ongoing media reception. An on-line judgment can turn into a memory-based judgment and vice versa. For instance, an individual has a pro-attitude (e.g., in a referendum) both at the beginning and at the end of a campaign. However, at the beginning she holds a memorybased judgment and at the end an on-line. This change in judgment type is again explained by alterations in the judgment formation goal. However, although judgments can vary in their type, this does not neces- 
sarily mean that they vary in their direction (i.e., pro or contra the referendum). The direction of a judgment (i.e., the judgment change) depends on alterations in media framing.

\section{Proposition 7}

For memory-based judgments, the recency, frequency and consonance of information predicts the direction of the judgment. As psychological research asserts (Higgins, 1996), constructs are more accessible when they have been recently or frequently activated. When a construct is activated, it has some residual excitation that can fade over time. Therefore, the shorter the time between a construct's last activation and judgment formation, the higher the likelihood that a construct will be accessed later on (Price and Tewksbury, 1997). Furthermore, when a construct is repeatedly activated, its accessibility will also increase. Beside the recency and frequency of activation, there is a third factor that can increase the accessibility of a construct: its consonance. This factor is especially crucial for media effects research, because news stories tend to provide conflicting or different views during a period of media coverage. That means a construct may recently and frequently occur, however, as long as there are other conflicting recently and frequently occurring considerations in media coverage, the construct's accessibility will be suppressed (for a similar argument see B. Scheufele, 2004). This line of reasoning is supported by an impressing recent study (Peter, 2004) which shows that consonant media coverage leads to stronger media effects. On the basis of this theorizing, we can predict that (memory-based) individuals will access those frames that have occurred recently, frequently and consonantly.

\section{Proposition 8}

In contrast, we can predict for the on-line model that recency, frequency and consonance of activation may play a minor role in predicting the direction of the judgment. Consistent with the psychological literature previously discussed (Hastie and Park, 1986), we can expect a weak relationship between the judgment and the information that people are able to recall. In line with this thinking, there should be an impact of those media frames encountered early in the temporal sequence of message presentation (Hamilton et al., 1999). Moreover, it can be assumed that individuals who form on-line judgments are more likely to be influenced by strong arguments. However, argument quality should be less important to individuals that compute memory-based judgments, because unelaborated encoding can increase the persuasive impact of weak arguments (Mackie and Asuncion, 1990). 


\section{Phases of framing effects}

Our model tries to distinguish two general phases of framing effects. The first phase describes the situation when individuals are confronted with a new issue or new evaluation object. No prior attitude is available at that point in time. The crucial question for the first phase is to describe under which circumstances news framing will lead to an on-line or to a memory-based route. For the second phase, the aim is to predict under which circumstances both judgmental types are likely to change or to remain constant. This means that a prior judgment exists or has been reported before. As should be apparent, both phases correspond to different time-points in the course of media effects. Phase one describes the creation of a new judgment, and phase two the change or stability of this judgment. In the course of the following discussion, the usefulness of distinguishing between these two phases of framing effects will become apparent.

\section{Phase 1: Confrontation with new information}

As stated above, a major objective of this model is to clarify under which conditions on-line and memory-based judgments are likely to be formed. We find several answers to this question in the research literature. First of all, there are grounds to believe that people may differ in their general tendency to perform on-line or memory-based judgments (Matthes et al., 2007). As Druckman and Nelson (2003) argue, the personality construct 'need to evaluate' (Jarvis and Petty, 1996) may play a crucial role in this context. Compared to individuals with a low need to evaluate, individuals high in need to evaluate create more spontaneous evaluative thoughts, they react more quickly to evaluative congruent words in a priming task, and they have stronger object-evaluation associations (Tormala and Petty, 2001). In an interesting experimental study, Tormala and Petty (2001) showed that high need to evaluate individuals were more inclined to form on-line attitudes than low need to evaluate individuals (for similar results Matthes et al., 2007; Druckman and Nelson, 2003). Obviously, we can expect robust interindividual differences in the extent to which individuals are motivated to form an on-line judgment. Second, we can find hints in the research literature that on-line reasoning is more likely to occur under conditions of high motivation (Lavine, 2002; Mackie and Asuncion, 1990; McGraw et al., 2003). In other words, the on-line model emphasizes the idea of a more goal-directed information processor. In order to form an on-line judgment, individuals need to have the willingness to attend to media coverage. But where does this motivation come from? As Lavine (2002) has recently argued, on-line 
judgments are more likely when an issue occupies a center stage in media coverage: " $[\mathrm{M}]$ edia attention to an issue increases citizens' motivation to think about and to elaborate on the issue, to form an opinion on the issue, and to discuss the issue with others in their social networks" (Lavine, 2002: 238). In essence, this idea corresponds to the basic agenda setting hypothesis. Numerous empirical studies provide clear evidence that, first and foremost, media salience (i. e., frequency and prominence of coverage) enhances the relevance people attach to issues (for a review see McCombs, 2004). Although there is a vast amount of other contingent conditions for agenda setting effects, media salience has an undeniable impact on issue relevance. We know from more than 35 years of agenda setting research that this perceived issue relevance, in turn, fosters the need for orientation toward an issue (Matthes, 2006; McCombs, 2004; Weaver, 1980). The need for orientation refers to the tendency of an individual to seek information about an issue in the news media. Rooted in the tradition of the uses and gratification approach, the need for orientation is a core concept in agenda setting research, and its impact on media effects has been demonstrated in several studies (e. g., McCombs, 2004; Weaver, 1980).

To sum up with an example, when there is an intensive coverage on the issue terrorism, it is more likely that people find this issue to be relevant. In turn, this triggers their need for orientation, which, eventually, fosters their motivation to form a judgment about that issue. Conceptually, we understand on-line and memory-based judgments as two different routes of judgment formation that can occur at the first phase of framing effects.

So far, we have explained the conditions that help to predict the type of judgment. However, as explained in proposition six, the direction of a judgment depends on the media's framing of an issue. For memorybased ones, the direction can be explained by the recency, the frequency and the consonance of frames. In other words, the most recent, frequent and consonant frames determine the direction of the memory-based judgment. Conversely, the primacy of information and argument strength shall be the major predictors for the direction of an on-line judgment.

Figure 1 summarizes the foregoing ideas about which factors contribute to the formation of an on-line or a memory-based judgment. The motivation to form an on-line judgment should generally be higher for people with a high need to evaluate compared to individuals with a low need to evaluate. Beside that, it can be assumed that prominent and frequent media coverage enhances the relevance people attach to issues. This enhanced relevance, in turn, impacts individuals' need for orientation. As a consequence, individuals with a high need for orientation will develop the motivational goal to form a judgment about that issue 


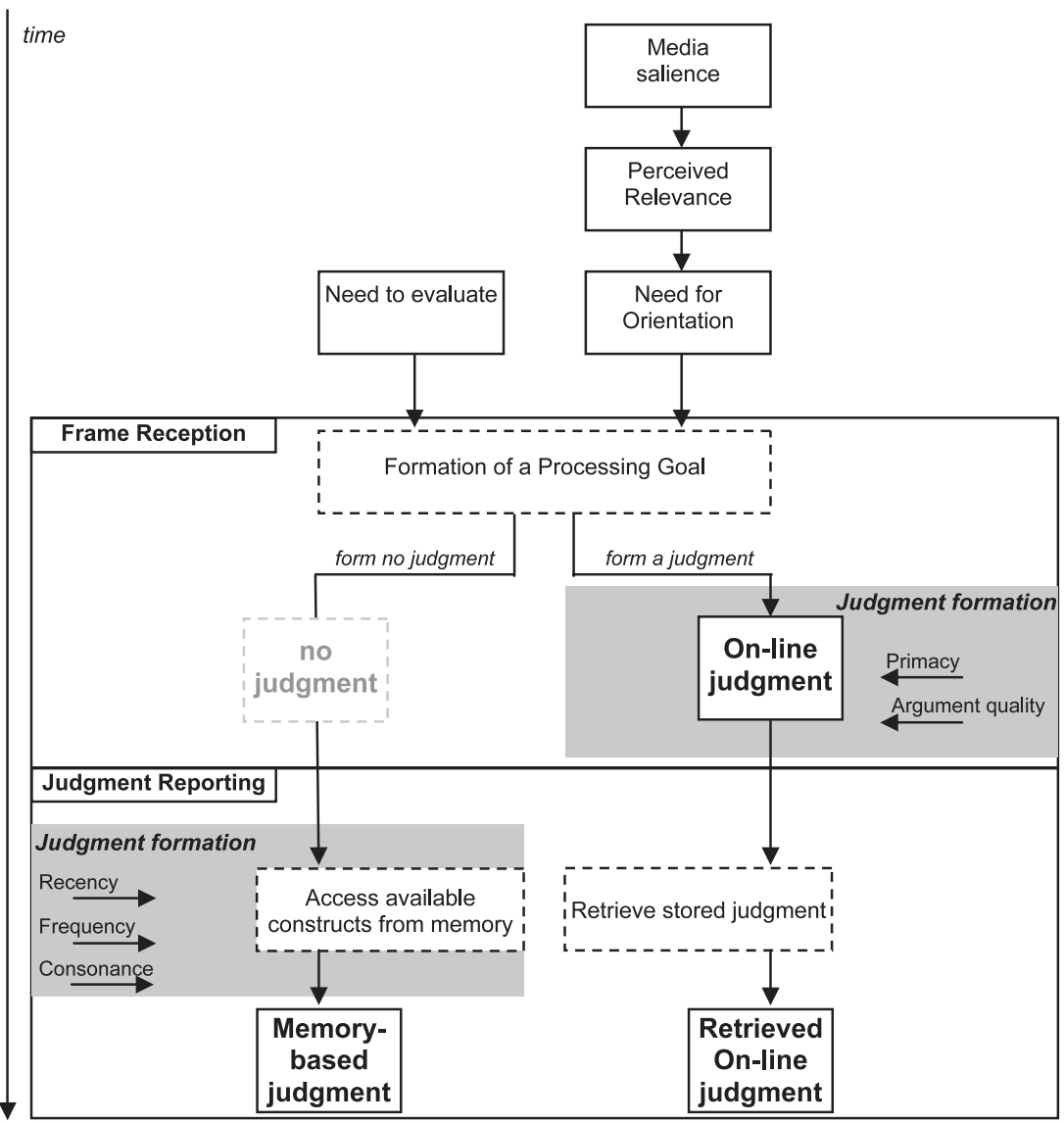

Figure 1. First phase.

whereas people with a low need for orientation prefer not to form a judgment. Now, when a judgment is called for, individuals with an online judgment can report a previously stored judgment, and individuals with a memory-based judgment search their memory for information in order to form an opinion. In the following section, we try to deliver some first insights about the consequences of on-line and memory-based judgments in terms of their susceptibility to change.

\section{Phase 2: Change or stability of on-line and memory-based judgments}

The second phase of framing effects describes how existing on-line or memory-based judgments are changed in the course of ongoing media 
coverage. In general, on-line judgments should produce higher judgment stability, and thus, a greater resistance to change (Hamilton et al., 1999). However, we know from social psychology that - depending on the circumstances - it is indeed possible that such judgments can be changed (e. g., Wilson, Lindsey, and Schooler, 2000). Thus, we assume that both memory-based and on-line judgments can be changed. In terms of observed media effects, the crucial questions are a) whether there is a change of the type of judgment, and b) whether there is a change in the direction of the judgment. A change in the type of judgment refers to the change of an on-line judgment into a memory-based one and vice versa. A change of the judgment direction, however, refers to the direction of the evaluation (i. e., pro or contra). A prerequisite for a change in judgment direction is a change in media framing. A prerequisite for a change in judgment type is a change in processing motivation.

In this paper, we wish to suggest that changes in judgment formation goals on the one hand and changes in the media's framing on the other hand can lead to six different routes at the second phase of framing effects: maintenance, readjustment, crystallization, inoculation, persuasion, and attenuation. The first three of them describe the change or stability of a memory-based judgment; the other three describe the change or stability of an on-line judgment.

Let's start with an initial memory-based judgment: In order to predict the change or the stability of a memory-based judgment the crucial point is whether the original judgment formation goal has altered or not. As we have explained before, people with a memory-based judgment tend to have a low need for orientation, and therefore, they develop the goal not to form a judgment during message reception. When there is no change in this need for orientation, the judgment formation goal will stay the same. This means, individuals will still not form judgments during ongoing media reception, but will again compute a judgment whenever needed on the basis of what they can remember.

To give an example, an individual receives a human-interest frame about a local issue at the first phase. Due to lack of interest in that issue, she will form no judgment. When she is asked about that issue, she will compute a judgment on whatever comes to mind at that time. When there is no increase in interest for phase two, the judgment formation goal will not change. Therefore, when asked at a later point in time, she will again base her judgment on accessible information. In sum, there is no change of judgment type (memory-based in both phases). However, although there is no change in judgment type, there could be a change in judgment direction. In principle, we can distinguish between two scenarios: First, when media framing does not change, individuals will most likely retrieve the same information as before, simply because they re- 
member the same media frames as before. Or, as Wilson and Hodges (1992) put it: "Stable attitudes are those with stable contexts". Therefore, we should expect no change in the overall judgment and in the constructs individuals can remember. We call this a maintenance effect, because the judgment direction will remain equal or unaltered. Second, when media framing does change, that means that other frames are reported frequently, recently or consonantly, individuals are likely to draw on these frames when computing a judgment. This is what we call a readjustment effect. The readjustment effect is completely in line with the frequently observed temporal instability of policy judgments (Lavine, 2002): When other constructs are made accessible by the media's framing, other judgments will follow (Zaller, 1992).

The third route is called crystallization. By crystallization, we mean the process of turning a memory-based judgment into an on-line judgment. A prerequisite for this to occur is a change of the initially established judgment formation goal. In other words, although individuals did not want to form a judgment at an earlier point in time, they now have a heightened need for orientation and strive to have a substantive judgment. Previous scholarship explains that this is most likely to happen when there is an increase in the perceived relevance of an issue. This in turn, will lead to an increase in individuals' need for orientation. As a consequence, individuals with a heightened need for orientation will now make up their minds and try to form an on-line judgment during message reception. As memory judgments tend to be unstable and cannot be stored and retrieved, this new on-line judgment should be largely independent from the previous memory-based judgment. We can expect those bits of information to be crucial for the judgment that are encountered early in the temporal sequence of message presentation in phase two (primacy effect). Moreover, argument quality should play a major role in determining the final judgment (Mackie and Asuncion, 1990). An example for crystallization would be if a person has no motivation to form a judgment at the beginning of a political campaign (memorybased judgment at phase one), however, but would develop a judgment formation goal at the end of a campaign. To the extent to which the judgment formation goal arises, this person would now listen to media coverage and built up a new on-line judgment that is largely independent from the earlier memory-based one.

So far, we have theoretically defined three types of framing effects: maintenance, readjustment and crystallization. What these three have in common is that they are originated from a memory-based judgment. Two of them, maintenance and readjustment, can still be regarded as memory-based. The crystallization effect, however, embodies a newly formed on-line judgment. 
The other three types concern the change or stability of an initial online judgment. In essence, these on-line judgments tend to be more stable and more resistant to change (Hamilton et al., 1999). As seen in the previous achievements in the field of social psychology, much scholarship has dealt with the topic of how people are persuaded by newly emerging or counter attitudinal information. The present paper attempts to build upon this previous research simply by differentiating between several hypothetical routes for a judgment change. As with an initial memory-based judgment, the crucial question is again whether or not people change their judgment formation goal in the course of ongoing media coverage. When individuals maintain their judgment formation goal, an on-line judgment will continue to exist, and will be highly accessible. As a consequence, we know since Klapper's (1960) seminal ideas that people prefer to expose themselves to information consonant with their own views, and thus, any new information will be biased in the direction of the initial on-line judgment (Klapper, 1960). Or, as RoskosEwoldsen (1997: 196) puts it: "When individuals have highly accessible attitudes, they are likely to process information in a biased manner, which will make any attempts at persuasion difficult". This results in a quite stable judgment; media coverage can probably exert no influence whatsoever. We call this phenomenon inoculation. In other words, inoculation describes a phenomenon where a once established on-line judgment remains stable over time. The type and the direction of the judgment do not change. To give an example, people build a first impression about a candidate, and this impression will be long lasting and can be retrieved easily. Thus, there is no change in judgment direction. Strictly speaking, inoculation cannot be regarded as an effect, but rather as a non-effect or resistance effect (see also Festinger and Maccoby, 1964; McGuire, 1964; Wegener, Petty, Smoak, and Fabrigar, 2004).

However, the theoretically more challenging question is how does one change a highly accessible on-line judgment? Put differently, when the judgment formation goal does not change and an already established online judgment remains highly accessible, how can such a judgment change at all? The crux of the homeostatic nature of on-line judgments is that they protect themselves; any threat of counter attitudinal arguments seems to be nipped in the bud. Moreover, on-line judgments tend to be activated automatically by the mere presentation of the attitude object (Fazio, 2000). Roskos-Ewoldsen (1997) explains that, however, the attempt to change highly accessible attitudes may not be as hopeless as resistance studies would seem to suggest. He asserts that accessible attitudes will most likely lead to biased processing when the presented information is ambiguous and open to multiple considerations: "When information is less ambiguous, accessible attitudes appear to exert propor- 
tionately less influence" (Roskos-Ewoldsen, 1997: 196). This argument is aided greatly by a large body of research about the factors that constitute an effective persuasive message. First and foremost, there are reasons to believe that the repeated exposure to extremely strong counter attitudinal arguments can result in persuasion (Mackie and Asuncion, 1990; Petty and Cacioppo, 1986). In addition, when people are highly motivated and are given sufficient opportunity, they can override their existing attitude. We can find a similar reasoning in the "model of dual attitudes" proposed by Wilson et al. (2000). The authors describe the processes of motivated and automatic overriding that can be quite enlightening in this context. Motivated overriding occurs when individuals view their prior attitude as illegitimate and unwanted, and thus, they are motivated to replace this attitude with a new attitude. This overriding requires effort and capacity; however, the so called automatic overriding does not. One example of automatic overriding is an attitude change resulting from introspection about the reasons for one's judgment. This idea is similar to the cognitive response approach in persuasion research (Petty, Ostrom and Brock, 1981). In short, according to the cognitive response approach, individuals generate thoughts or cognitive responses when being exposed to a message. The more these cognitive responses contradict their prior attitude, the greater the attitude change should be (Petty et al., 1981).

Moreover, other studies in the dissonance paradigm have shown that inducing people to perform counter attitudinal behavior for low, external justification can lead to an attitude change in the direction of the behavior (e. g., Festinger and Carsmith, 1959).

In sum, the sheer complexity of the persuasion process provides a vast array of possible intervening variables which are clearly beyond the scope of this paper. Taken together, all examples described above lead us to suggest a fifth route of framing effects: the (classical) persuasion effect. As the previous review has demonstrated, there are grounds to believe that media framing can exert an impact on accessible attitudes under some circumstances, although such an effect might be rather the exception than the rule. In essence, the most important predictor for an attitude change seems to be the strength of counter arguments put forward in the media's framing (Mackie and Asuncion, 1990; Petty and Cacioppo, 1986).

The last route we like to suggest is called attenuation. Attenuation refers to a process where an initial on-line judgment will turn into a memory-based judgment. As should be apparent now, this is most likely to occur when there is a decrease in need for orientation in a way that people no longer have the desire to form a judgment about an issue. For instance, when media coverage about an issue diminishes, the judgment 
will be less likely to be retrieved. This can be explained by the activation potential of stored on-line judgments: When the activation potential drops under a threshold level its accessibility will decrease (Price and Tewksbury, 1997). Interestingly, the same factors contribute to the accessibility of an attitude as to the accessibility of lower-order constructs: the recency, frequency and consonance of prior activation. Therefore, when an on-line judgment is not recently, frequently or consonantly activated, it will attenuate, that means, it will not be retrievable at a later point time (Fazio, 2000). As a result, when a judgment is called for, individuals cannot retrieve the on-line judgment anymore, and they are thus likely to construct a new one on the basis of accessible information in memory (i.e., a new memorybased judgment). This new judgment, in turn, will be influenced by the recency, frequency and consonance of media framing at phase two. Attenuation might occur when issues fade in their popularity. A prominent example for this idea is the issue-attention-cycle that states that issues suddenly leap into prominence, remain there for a short time, and then gradually fade from the center of public attention.

Figure 2 summarizes the two phases of framing effects. The depicted model attempts to highlight how on-line and memory-based judgments are created (first phase) and how are they changed (second phase). As such, it provides a holistic view on the relationships among the variables leading to the prediction of framing effects. The upper part of the model shows the formation of on-line and memory-based judgments. Although simplified, this part corresponds to figure 1 discussed above. We predict that on-line judgments are likely to be formed under conditions of a high need for orientation, whereas memory-based judgments tend to be the result of a low level of need for orientation. The main body of the model tries to describe how the media's framing changes memory-based and on-line judgments. For that purpose, we posit six routes leading to six different effects.

How can these six different effects be distinguished empirically? As we have explained in proposition five, the major difference between an online and a memory-based judgment is judgment strength. Moreover, we can differentiate these six effects in regard to the direction of the final judgment, i.e., whether or not the judgment changes from phase one to phase two.

Table 1 lists the six types and their main characteristics. Maintenance means that an equal memory-based judgment will be created at a later point in time. The prerequisite for maintenance is that the media's framing does not change. Consequently, there will be no attitude change. Just like maintenance, readjustment is memory-based at both time-points. The accessible information, however, changes in the course of media coverage as different frames come up in different times of the debate. 


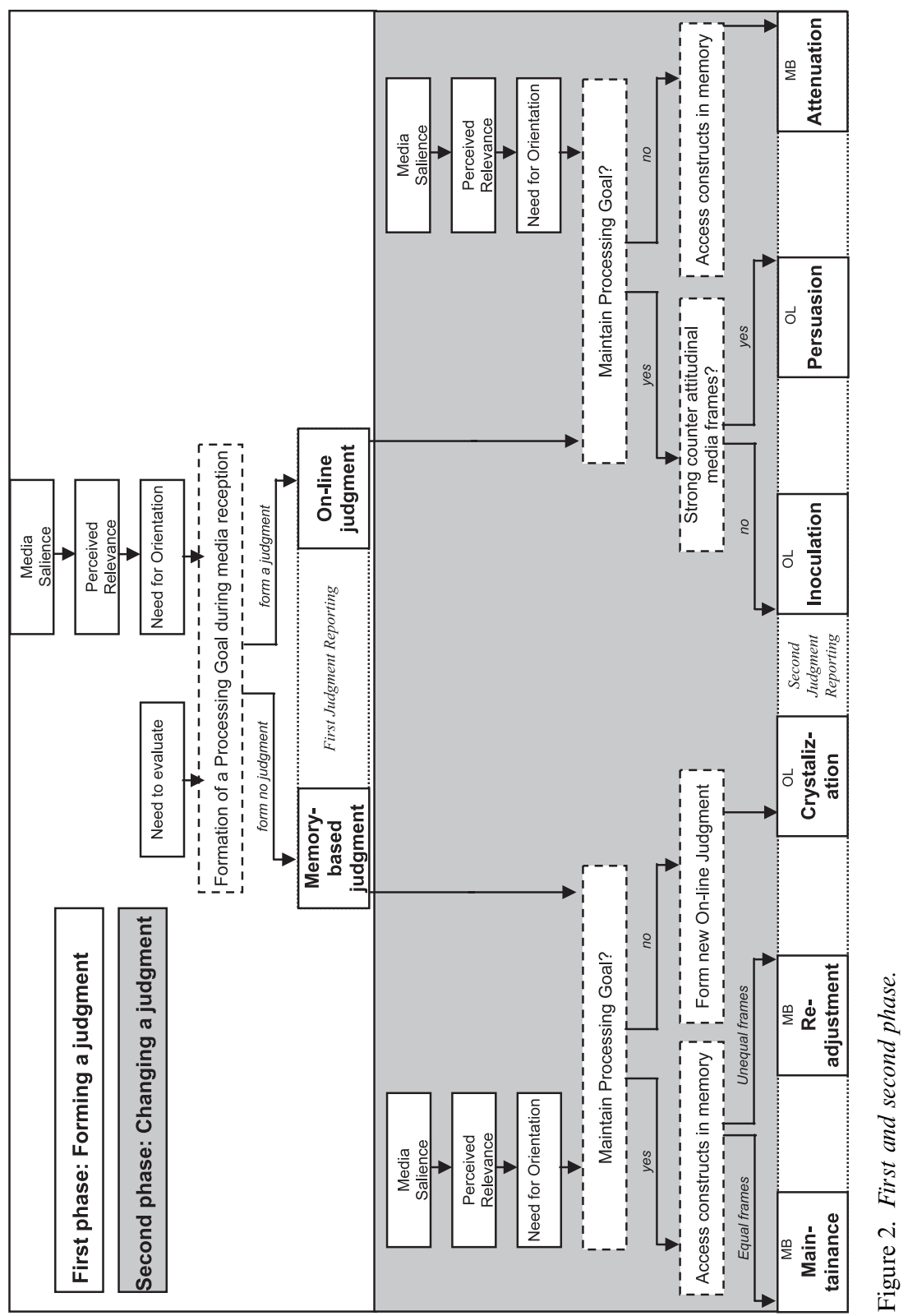


Table 1. Main characteristics of the six routes.

\begin{tabular}{|c|c|c|c|c|c|c|c|}
\hline & \multicolumn{2}{|l|}{ Phase 1} & \multicolumn{2}{|c|}{ Predictor variables } & \multicolumn{2}{|l|}{ Phase 2} & \multirow{2}{*}{$\begin{array}{l}\text { Judgment } \\
\text { direction }\end{array}$} \\
\hline & $\begin{array}{l}\text { Type of } \\
\text { judgment }\end{array}$ & $\begin{array}{l}\text { Judgment } \\
\text { Strength }\end{array}$ & $\begin{array}{l}\text { Change of } \\
\text { judgment } \\
\text { formation } \\
\text { goal }\end{array}$ & $\begin{array}{l}\text { Change of } \\
\text { media } \\
\text { framing }\end{array}$ & $\begin{array}{l}\text { Type of } \\
\text { judgment }\end{array}$ & $\begin{array}{l}\text { Judgment } \\
\text { strength }\end{array}$ & \\
\hline Maintenance & $\begin{array}{l}\text { memory- } \\
\text { based }\end{array}$ & low & no & no & $\begin{array}{l}\text { memory- } \\
\text { based }\end{array}$ & low & no \\
\hline Readjustment & $\begin{array}{l}\text { memory- } \\
\text { based }\end{array}$ & low & no & yes & $\begin{array}{l}\text { memory- } \\
\text { based }\end{array}$ & low & yes \\
\hline Crystallization & $\begin{array}{l}\text { memory- } \\
\text { based }\end{array}$ & low & yes & yes/no & on-line & high & yes/no \\
\hline Inoculation & on-line & high & no & no & on-line & very high & no \\
\hline Persuasion & on-line & high & no & yes & on-line & high & yes \\
\hline Attenuation & on-line & high & yes & yes/no & $\begin{array}{l}\text { memory- } \\
\text { based }\end{array}$ & low & yes/no \\
\hline
\end{tabular}

Therefore, readjustment results in a different judgment. Both types can be distinguished by measuring media content, judgment type, and judgment direction. Again, maintenance and readjustment do not differ in judgment type (i.e., they are memory-based), but they do differ in judgment direction.

The third route, crystallization, characterizes the change of a memorybased judgment into an on-line judgment. This can occur due to an increase in judgment formation motivation. As Table 1 shows, the change of the judgment from phase one to phase two depends on the media content. When the media's framing changes, there will also be a change of judgment direction; however, when the media's framing remains constant, judgment direction will not be changed. The crucial point of crystallization, however, is that judgment strength should be higher for phase two compared to phase one.

As known from classic literature on persuasion, inoculation describes the quite likely process that on-line judgments remain stable over time. Such judgments should be held with a very high attitude strength. On the contrary, persuasion is a phenomenon where a previously stored online judgment is changed, mainly because of strong counter attitudinal arguments. In both cases, the type of judgment does not change, but in case of persuasion, the direction of the judgment does.

Finally, by attenuation we mean the fading of an on-line judgment leading to a memory-based judgment at a later point in time. As the online judgment at phase one ceases to exist, the new memory-based judgment will depend on the media's framing in the second phase. This can be similar or contrary to phase one, and hence, the direction of the judgment can change or not. 


\section{Discussion}

This article hopes to make a theoretical contribution to framing theory by introducing means to distinguish several routes for framing effects. We have begun by addressing how framing effects have been theoretically explained in previous scholarship. In doing so, we have outlined that most theoretical models clearly build on a memory-based account of judgment formation. However, as we have learned from numerous studies in judgment and attitude research, a model solely focusing on memory-based judgments seems to offer a limited account in explaining the full process of opinion formation. Therefore, we have suggested that theorizing about framing effects should embrace both, memory-based and on-line mechanisms. It is to stress that we do not find previous models, such as Price and Tewksbury's, to be incompatible with our reasoning. In contrast, we believe that our account can be an extension of highly valuable previous theorizing, not a substitution. Hence, our model does not deny the notion of accessibility in judgment formation. But it does go 'beyond accessibility' in so far as it introduces the idea that framing effects can emerge on-line during initial message processing.

We have proposed a model that distinguishes between two phases of framing effects: Along the first phase, the media's framing contributes to the formation of an on-line or a memory-based judgment. The second phase describes six hypothetical routes for the stability or the change of these judgments. Moreover, we have tried to extract the predictors for each of those paths. We distinguished between those routes for reasons of theoretical clarity, and because we wanted to provide framing scholars with an organizing scheme for the empirical literature. However, in our view the model is more than a recapitulation of the literature. It is an extension of previous theorizing in framing research as it links framing effects with a psychological theory. This model allows assumptions about the type, the stability, and the direction of judgments.

Although the model provides a general account on judgment formation, the focus is clearly on framing effects. However, as stated above, the model does not allow deriving hypotheses about specific media frames. Instead, it incorporates broad characteristics of media frames, such as whether the framing of an issue changes, and how recently, frequently, and consonantly an issue is framed. In essence, framing is treated as the major variable that helps to explain changes in the direction of existing judgments. Changes in the type of judgments, however, are mainly explained by psychological variables such as need for orientation.

Of course, we must acknowledge that it is not a simple matter to distinguish between several routes of framing effects and that there is 
more empirical support for some routes than for others. As has often been noted, no single theoretical integration can be considered as definite with respect to all relevant scientific questions. Given this caveat, we nevertheless see several strengths of our endeavor. We hope that our typology is useful as a first organizing scheme which can be illuminating for future framing research. Now, having this organizing scheme, there seem to appear some pressing issues. To begin with, we as framing scholars should pay more attention to the exact nature of framing effects. For our understanding of the longevity and robustness of such effects, it is crucial to know whether we speak of an on-line or a memory-based judgment. Incorporating these insights into framing theory does not only allow us to better predict the effects of mass media's framing on the overall judgment, we can also draw conclusions about how judgment and memory for judgment related information will correlate. Therefore, our model might bring framing scholars to deeper insights about how individuals draw on information presented in the mass media in order to compute a judgment. Moreover, in looking at the nature of framing effects, we should also widen our comprehension about the time a judgment is formed. This refers to the ultimate question whether judgments are formed during initial message exposure or later on when a judgment is called for. Beside that, we have enumerated some variables that are thought to predict the different routes for on-line and memory-based judgments. Knowing which variables lead to which framing effects might also equip us to better predict the audience's framing of an issue in a long-term perspective.

However, we have only reached some preliminary conclusions about when one or when the other effect is more likely to occur, and thus we are far from having a comprehensive analysis of the full range of framing effects. Hence, it goes without saying that there are a number of limitations to the present model which are worthy of careful consideration. Among these, perhaps the most critical is the eclectic nature of our model. We have tried to summarize previous achievements from different fields of scholarship, such as framing effects research, political science, cognitive psychology, or the social psychology of persuasion. Conceptually, we believe that this is a worthwhile effort because our understanding of media effects in general lives from an understanding of individual psychological processes. However, one could object that such an eclectic approach cannot do justice to the richness of individual perspectives on framing effects. In defense of the generality of the ideas presented in this paper, we have cited a number of studies that have obtained results in line with our reasoning. However, we have to admit that there must remain some blind spots. At present, we are not aware of a single study testing our conjectures in unequivocal ways. As a consequence, we must 
conduct more empirical research to explore every phenomenon in more detail.

In this context, especially longitudinal studies are urgently needed. Only when applying a long-term perspective, are we able to observe how and when memory-based or on-line judgments are likely to be created, and how these judgments can be changed in the course of ongoing media coverage. For instance, during political campaigns it is crucial to know whether people form an on-line or a memory-based judgment at the beginning of the campaign. Knowing that, we can predict different media effects for different individuals. To give an example, a panel study could diagnose the judgment type in the several waves and measure predictors such as need for orientation. In addition to that, media frames should be accessed in a content analysis. When combing the two data sources, we can observe how changes in media framing impact judgments for individuals with different judgments types and different judgment formation goals. Ideally, the model allows a set of predictions about when framing effects shall occur. Theoretically, the model could be helpful both for issues with prior judgments and for issues without prior judgments. When people have not formed an opinion about a newly emerging issue, phase one of the model helps to explain whether an on-line or a memory-based judgment will be formed. However, when we deal with an established issue phase two of the model could explain how already existing on-line and memory-based judgments are likely to change. For all these research questions, we hope that this theoretical paper provides a first step on this promising avenue of this research.

\section{References}

Cappella, J. N. and Jamieson, K. H. (1997). Spiral of cynicism. The press and the public good. New York, Oxford: Oxford University Press.

Chaiken, S. and Trope, Y. (Eds.) (1999). Dual process theories in social psychology. New York: Guilford.

Druckman, J. N. and Lupia, A. (2000). Preference formation. Annual Review of Political Science, 3, 1-24

Druckman, J. N. and Nelson, K. R. (2003). Framing and deliberation: How citizens' conversations limit elite influence. American Journal of Political Science, 47, $729-745$.

Druckman, J. N. (2002). The implications of framing effects for citizen competence. Political Behavior, 23(3), 225-256.

Entman, R. M. (1993). Framing: toward clarification of a fractured paradigm. Journal of Communication, 43(4), 51-58.

Fazio, R. H. (2000). Accessible attitudes as tools for object appraisal: Their costs and benefits. In G. Maio and J. Olson (Eds.), Why we evaluate: Functions of attitudes (pp. 1-36). Mahwah, NJ: Erlbaum.

Festinger, L. and Carlsmith, J. M. (1959). Cognitive consequences of forced compliance. Journal of Abnormal and Social Psychology, 58, 203-211. 
Festinger, L. and Maccoby, N. (1964). On resistance to persuasive communications. Journal of Abnormal and Social Psychology, 68, 359-366.

Gross, K. and D'Ambrosio, L. (2004). Framing emotional response. Political Psychology, 25(1), 1-29.

Hamilton, D. L., Sherman, S. J., and Maddox, K. B. (1999). Dualities and continua: Implications for understanding perceptions of persons and groups. In S. Chaiken, and Y. Trope (Eds.), Dual process theories in social psychology (pp. 606-626). New York: Guilford.

Hastie, R. and Park, B. (1986). The relationship between memory and judgment depends on whether the judgment task is memory-based or on-line. Psychological Review, 93(3), 258-268.

Higgins, E. T. (1996). Knowledge activation: Accessibility, applicability, and salience. In E. T. Higgins, and A. W. Kruglanski (Eds.), Social psychology: Handbook of basic principles (pp. 133-168). New York: Guilford.

Huang, L. (2000). Examining candidate information search processes: The impact of processing goals and sophistication. Journal of Communication, 50(1), 93-114.

Igartua, J. J., Cheng, L., and Muñiz, C. (2005). Framing Latin America in the Spanish press. A cooled down friendship between two fraternal lands. Communications, 30(3), 359-372.

Iyengar, S. (1991). Is anyone responsible? How television frames political issues. Chicago, IL: University of California Press.

Jarvis, W. B. G. and Petty, R. E. (1996). The need to evaluate. Journal of Personality and Social Psychology, 70(3), 172-194.

Kahneman, D. and Tversky, A. (1984). Choices, values, and frames. American Psychologist, 38, 341-350.

Klapper, J. T. (1960). The effects of mass communication. Glenco, IL: The Free Press.

Lavine, H. (2002). On-Line versus memory-based process models of political evaluation. In K. R. Monroe (Ed.), Political Psychology (pp. 225-274). Mahwah, NJ: Erlbaum.

Lippman, W. (1922). Public opinion. New York: Free Press.

Lodge, M., McGraw, K. M., and Stroh, P. (1989). An impression-driven model of candidate evaluation. The American Political Science Review, 83(2), 399-419.

Mackie, D. M. and Asuncion, A. G. (1990). On-line and memory-based modification of attitudes: Determinants of message recall-attitude change correspondence. Journal of Personality and Social Psychology, 59(1), 5-16.

Matthes, J. (2006). The need for orientation. Revising and validating a classic concept. International Journal of Public Opinion Research, 18(4), 422-444.

Matthes, J., Wirth, W., and Schemer, C. (2007). Measuring the Unmeasurable? Toward operationalizing on-line and memory-based political judgments in surveys. International Journal of Public Opinion Research, 19(2) (forthcoming).

McCombs, M. E. (2004). Setting the agenda: The news media and public opinion. Malden, MA: Polity Press.

McCombs, M. E. and Ghanem, S. I. (2001). The convergence of agenda setting and framing. In S. D. Reese, O. H. Gandy, and A. E Grant (Eds.), Framing public life: perspectives of media and our understanding of the social world (pp. 67-81). Mahwah, NJ: Erlbaum.

McGraw, K. (2000). Contributions of the cognitive approach to Political Psychology. Political Psychology, 21(4), 805-832.

McGraw, K. M., Hasecke, E., Conger, K. (2003). Ambivalence, uncertainty, and processes of candidate evaluation. Political Psychology, 24(3), 421-448.

McGuire, W. J. (1964). Inducing resistance to persuasion. In L. Berkowitz (Ed.), Advances in experimental social psychology (Vol. 1, pp. 192-229), New York: McGraw-Hill. 
Nabi, R. L. (2003). The framing effects of emotion: Can discrete emotions influence information recall and policy preference? Communication Research, 30(2), 224247.

Nelson, T. E., Clawson, A., and Oxley, Z. M. (1997). Toward a psychology of framing effects. Political Behavior, 19(3), 221-246.

Peter, J. (2004). Our long 'return to the concept of powerful mass media' - A crossnational comparative investigation of the effects of consonant media coverage. International Journal of Public Opinion Research, 16(2), 144-168.

Petty, R. E. and Cacioppo, J. T. (1986). The elaboration likelihood model of persuasion. New York: Academic Press.

Petty, R. E., Ostrom, T. M., and Brock, T. C. (1981). Cognitive responses in persuasion. Hillsdale, NJ: Erlbaum Associates.

Price, V. and Tewksbury, D. (1997). News values and public opinion: A theoretical account of media priming and framing. In G. Barnett and F. J. Boster (Eds.), Progresses in the Communication Sciences (pp. 173-212). Greenwich, CT: Ablex

Price, V., Tewksbury, D., and Powers, E. (1997). Switching trains of thought: The impact of news frames on reader's cognitive responses. Communication Research, 24(5), 481-506.

Roskos-Ewoldsen, D. R. (1997). Attitude accessibility and persuasion: Review and a transactive model. In B. R. Burleson and A. W. Kunkel (Eds.), Communication Yearbook (pp. 185-225), Thousand Oaks: Sage.

Scheufele, B. (2004). Framing-effects approach: A theoretical and methodological critique. Communications, 29(4), 401-428.

Scheufele, B. (2006). Frames, schemata and news reporting. Communications, 31(1), $65-83$.

Scheufele, D. A. (2000). Agenda-setting, priming, and framing revisited: Another look at cognitive effects of political communication. Mass Communication and Society, $3(2), 297-316$.

Schwarz, N. (2000). Social judgment and attitudes: warmer, more social, and less conscious. European Journal of Social Psychology, 30, 149-176.

Schwarz, N. (in press). Attitude construction: Evaluation in context. Social Cognition.

Shen, F. (2004). Chronic accessibility and individual cognitions: Examining the effects of message frames in political advertisements. Journal of Communication, 54(1), $123-137$.

Shrum, L. J. (2004). The cognitive processes underlying cultivation effects are a function of whether the judgments are on-line or memory-based. Communications, 29(3), 327-344.

Tormala, Z. L. and Petty, R. E. (2001). On-line versus memory-based processing: The role of "need to evaluate" in person perception. Personality and Social Psychology Bulletin, 27(12), 1599-1622.

Valkenburg, P. M., Semetko, H. A., and de Vreese, C. H. (1999). The effects of news frames on readers' thoughts and recall. Communication Research, 26(5), 550-569.

Vreese, C. H., de (2004). The effects of strategic news on political cynicism, issue evaluations and policy support: A two-wave experiment. Mass Communication and Society, 7(2), 191-215.

Vreese, C. H., de and Boomgaarden, H. (2003). Valenced news frames and public support for the EU. Communications, 28(4), 361-381.

Weaver, D. H. (1980). Audience need for orientation and media effects. Communication Research, 3, 361-376.

Wegener, D. T., Petty, R. E., Smoak, N. D., and Fabrigar, L. R. (2004). Multiple routes to resisting attitude change. In E. S. Knowles and J. A. Linn (Eds.), Resistance and persuasion (pp. 13-38). Mahwah, NJ: Erlbaum. 


\section{Jörg Matthes}

Wilson, T. D. and Hodges, S. D. (1992). Attitudes as temporary constructions. In L. L Martin and A. Tesser (Eds.), The construction of social judgments (pp. 37-65). Hillsdale, NJ: Erlbaum.

Wilson, T. D., Lindsey, S., and Schooler, T. Y. (2000). A model of dual attitudes. Psychological Review, 107, 101-126.

Zaller, J. R. (1992). The nature and origins of mass opinion. Cambridge: Cambridge University Press. 